GUNNAR NIEMI

\title{
ON THE EXISTENCE OF A MODAL ANTINOMY
}

1. Our interest here is in the relations between the syntactical predicate '___ is true' and the syntactical modal predicates such as '___ is necessary', ‘ is possible', ' is contingent', and ' entails...' That there should be similarities between these predicates is not surprising since the modal predicates are so-called because they deal with what used to be known as 'modes' of truth. " synonymous with '___ is necessarily true'. is necessary', for example, is

The alleged pre-analytic view of the correct use of the predicate is true' is that we may accept all instances of the schema ' is true if and only if

It is well known that this naive view leads to antinomies, or contradictions. Restrictions against accepting all instances of (1.1) are necessary. In particular, certain self-referential sentences must not be allowed to occupy the place of the blank in schema (1.1).

By a syntactical view of modality is meant a view which holds that modal statements should be formed by attaching modal predicates such as " is necessary' to names of sentences. Non-syntactical views differ in that they allow modal statements to be formed by attaching operators such as 'It is necessary that' to sentences themselves.

Although it has often been claimed that there are philosophical advantages to adopting a syntactical view of modality (e.g. [7]), there has not been much interest in the construction of formal theories to represent syntactical modal statements. However, given a formal theory designed to represent non-syntactical modal statements, such as one of the familiar Lewis systems, we can easily construct an analogous syntactical theory. Corresponding to the non-syntactical modal statements which would be represented as instances of the Lewis schema ' $\square p \supset p$ ' we have the instances of the schema

If ' is necessary, then 
Modal schemata such as (1.2) can be taken as giving us rules for the correct use of the modal predicates. One of the main questions to be dealt with in this paper is whether schemata such as (1.2) are like schema (1.1) for the use of ' is true' in point of requiring restrictions against acceptance of certain self-referential instances. Since there are various formal modal theories, each providing us with different schemata, it is of course possible that some modal theories require restrictions while others do not.

In Part 2 of this paper we shall offer a description of an ordinary language modal antinomy analogous to the antinomy of the Liar. In Part 3 we shall describe a formal theory SM and certain extensions thereof in which modal antinomies could be represented if they existed. SM will have as theorems the syntactical counterparts of Lewis' S5. In Part 4 we shall outline a proof of the consistency of the relevant extensions of SM, thereby showing that there are no modal antinomies of the sort described in Part 2 for modal theories no stronger than Lewis' S5.

In Part 5 we shall discuss certain results by Richard Montague which might easily be interpreted as implying the existence of a modal antinomy. In Part 6 we shall give an explanation of why Montague's results do not in fact imply the existence of a modal antinomy of the sort described in Part 2.

2. The antinomy of the Liar is the best known of the antinomies resulting from unrestricted acceptance of the instances of schema (1.1). A particularly simple version of this antinomy may be stated as follows.

(2.1) The Antinomy of the Liar. A contradiction follows from the acceptance of all instances of the schema

(a) '_ ' is true if and only if

together with the empirical identity statement

(b) The 1st sentence on $B$ is 'The 1st sentence on $B$ is not true' (where ' $B$ ' is an abbreviation of a description which singles out a particular blackboard at a particular time).

The antinomy is easily demonstrated by taking as an instance of (a)

(2.2) 'The 1st sentence on $B$ is not true' is true if and only if the 1st sentence on $B$ is not true 
and making the identity substitution licensed by (b) to obtain

(2.3) The 1st sentence on $B$ is true if and only if the 1st sentence on $B$ is not true.

We shall take the following as our description of an ordinary language modal antinomy analogous to the antinomy of the Liar.

(2.4) The syntactical theory of modality $S$ has a modal antinomy $={ }_{\mathrm{df}}$. There could be a set of modal statements upon the blackboard $B$ such that a contradiction could be inferred from instances of theorems of $S$ together with the identity statements generated by the placement of sentences on $B$.

By a modal statement in (2.4) is meant a statement all of whose predicates are modal predicates.

The identity statements mentioned in (2.4) will be statements of the form 'The $n$th sentence on $B$ is " "' where the sentence which fills the blank is in fact the $n$th sentence on $B$. A particular configuration of chalk upon $B$ might yield the following identity statements.

The 1st sentence on $B$ is 'The 2 nd and 3 rd sentences on $B$ are necessary'

The 2 nd sentence on $B$ is 'The 2 nd sentence on $B$ entails the negation of the 1st sentence on $B^{\prime}$

The $3 \mathrm{rd}$ sentence on $B$ is 'The $3 \mathrm{rd}$ sentence on $B$ is not necessary'.

We allow $B$ to contain more than one sentence because we wish to allow for the possibility of a modal antinomy involving indirect selfreference, as well as for the possibility of one involving only direct selfreference. We say that a sentence is directly self-referential if it contains singular terms referring to itself and that it is indirectly self-referential if it contains only singular terms referring to other sentences which eventually refer back to the original sentence.

There is a well known antinomy involving " is true' which uses only indirect self-reference. This antinomy consists of a pair of sentences, the first of which asserts the truth of the second and the second of which denies the truth of the first.

3. The language for our theory SM is formed by adding the single quotation mark "' 'to an ordinary first-order language with identity. It 
will be convenient to suppose that this language has negation, disjunction, and existential quantification as primitive operations.

Since the single quotation mark is to simulate the behavior of quotation marks in English we adopt the formation rule

(3.1) If $A$ is a sentence, then $\left.\Gamma^{c} A^{\prime}\right\urcorner$ is a term.

To preserve the referential opacity of quotation marks we stipulate that those occurrences of terms which are proper parts of terms of the form $\Gamma^{-} A$ ' $\urcorner$ are bound occurrences. This stipulation will prevent identity substitutions within quoted expressions and also quantification into quoted expressions.

SM contains the following non-logical symbols.

Constants: ' $a$ ', ' $b$ ', ' $c$ ', ' $a_{1}{ }^{\prime}, \ldots$

A one-place predicate symbol: ' $N$ '

A one-place function symbol: 'neg'

A two-place function symbol: 'dis'

' $N$ ' is to represent ' is necessary'. 'neg' and 'dis' allow us to talk about negations and disjunctions of sentences, and hence of all truthfunctions. We can define the other modal predicates in terms of ' $N$ '. For example, we may use ' $P$ ' to represent " is possible' and ' $E$ ' to represent : entails...'. introducing these letters into our theory by way of the definitions

$$
\begin{aligned}
& (x)(P(x) \equiv \sim N(\operatorname{neg}(x))) \\
& (x)(y)(E(x, y) \equiv N(\operatorname{dis}(\operatorname{neg}(x), y)))
\end{aligned}
$$

All instances of the following schemata, where $A$ and $B$ are sentences, are non-logical axioms of SM.
(a) $N\left({ }^{\prime} A^{\prime}\right) \supset A$
(b) $N\left({ }^{\prime} A \supset B^{\prime}\right) \supset\left(N\left({ }^{\prime} A '\right) \supset N\left({ }^{\prime} B^{\prime}\right)\right)$
(c) $\sim N\left({ }^{\circ} A^{\prime}\right) \supset N\left({ }^{\circ} \sim N\left({ }^{\prime} A^{\prime}\right)^{\prime}\right)$
(d) $\operatorname{neg}\left({ }^{\circ} A^{\prime}\right)={ }^{\prime} \sim A^{\prime}$
(e) $\operatorname{dis}\left({ }^{\circ} A^{\prime},{ }^{\prime} B^{\prime}\right)={ }^{\prime} A \vee B^{\prime}$
(f) ' $A$ ' $\neq{ }^{\prime} B$ ', where $A$ and $B$ are different sentences.

SM allows the following inference. 
(3.5) From a sentence $A$ to infer $\left\ulcorner N\left({ }^{\prime} A^{\prime}\right)\right\urcorner$, provided that the sequence of expressions up to and including $A$ is such that the result of deleting some number, possibly zero, of these expressions is a proof of $A$ in SM.

The first three axioms of (3.4) together with the inference (3.5) are the syntactical analogues of a common axiomatization of Lewis' S5.

An I-extension of SM is a theory which results from SM by the addition of a finite number of non-logical axioms of the form $\left\ulcorner a_{i}={ }^{\prime} A_{i}{ }^{\prime}\right\urcorner$, where each $a_{i}$ is a distinct constant and each $A_{i}$ is a sentence, although not necessarily distinct. These non-logical axioms are known as the theory's I-axioms.

I-axioms are intended as representations of the identity statements generated by the arrangement of sentences on the blackboard $B$,-as described in Part 2. Corresponding to the identity statements in (2.5), for example, we have the I-extension whose I-axioms are

$$
\begin{aligned}
& a={ }^{`} N(b) \& N(c) ' \\
& b=` N(\operatorname{dis}(\operatorname{neg}(b), \operatorname{neg}(a))){ }^{\prime} \\
& c={ }^{\prime} \sim N(c)^{\prime}
\end{aligned}
$$

The cumbersome statement of the inference (3.5) is to prevent the application of the necessitation rule to I-axioms and expressions which depend on I-axioms. That the $n$th sentence on $B$ should be such-and-such is a contingent and not a necessary fact.

In the next part we shall outline a proof of the consistency of every I-extension of SM. This will show that there is no modal antinomy of the sort described in Part 2 for syntactical modal theories no more powerful than S5.

To illustrate the expressive power of SM we compare it to a similar theory ST intended as a formalization of a syntactical theory of truth. ST is like SM except for containing the one-place predicate symbol ' $T$ ' in place of ' $N$ ' and the instances of the single schema $\left\ulcorner T\left({ }^{\prime} A\right.\right.$ ') $\left.\equiv A\right\urcorner$ in place of the instances of the first three schemata of (3.4). ST omits the inference (3.5).

It is easy to show that the I-extensions of ST are not all consistent. Corresponding to the antinomy of the Liar is the I-extension whose only I-axiom is ' $a=$ ' $-T(a)$ '. A simple proof shows this theory to be inconsistent. 

(1) $a={ }^{\circ} \sim T(a)^{\prime}$
I-axiom
(2) $T\left({ }^{\circ} \sim T(a)^{\prime}\right) \equiv \sim T(a)$ axiom
(3) $T(a) \equiv \sim T(a)$
1,2

Corresponding to the antinomy in which a first sentence asserts the truth of a second while the second denies the truth of the first is the inconsistent I-extension of ST whose I-axioms are ' $a={ }^{\prime} T(b)$ '" and ' $b=$ $=$ ' $\sim T(a)^{\prime}$.
(1) $a=' T(b)$ '
I-axiom
(2) $b={ }^{\prime} \sim T(a)$ '
I-axiom
(3) $T\left({ }^{\prime} T(b)^{\prime}\right) \equiv T(b)$ axiom
(4) $T(a) \equiv T(b)$
1,3
(5) $T(a) \equiv T\left({ }^{6} \sim T(a)^{\prime}\right)$
2,4
(6) $T\left({ }^{\circ} \sim T(a)^{\prime}\right) \equiv \sim T(a)$ axiom
(7) $T(a) \equiv \sim T(a)$ 5,6

In discussing self-referential sentences Kaplan and Montague state the following ([2], p. 82), where their $\ulcorner\bar{S}\urcorner$, the standard name of $S$, can be identified with the $\Gamma$ ' $S$ ' 7 of SM.

... whenever we are given a formula $F$ whose sole free variable is ' $x$ ', we can find a sentence $S$ which is provably equivalent to $F(\bar{S})$, that is, the result of replacing in $F$ the variable ' $x$ ' by the standard name of $S$. The sentence $F(\bar{S})$ makes a certain assertion about the sentence $S$. Since $S$ is provably equivalent to $F(\bar{S}), S$ makes the same assertion about $S$, and thus is self-referential. Besides this method and its variants, no other precise ways of treating self-referential sentences are known to us.

All self-referential sentences of the sort described above are representable in I-extensions of SM. Let $F(x)$ be a formula of SM with ' $x$ ' as its only free variable. Consider the I-extension of SM which contains $\ulcorner a=F(a)\urcorner$ as an I-axiom. In this theory we have $\left\ulcorner F(a) \equiv F\left({ }^{(} F(a)^{\prime}\right)\right\urcorner$ as a theorem. Thus letting $\mathrm{S}$ be $\ulcorner F(a)\urcorner$ with its standard name $\left.\Gamma^{\circ} F(a){ }^{\prime}\right\urcorner$ we have the provable equivalence $\left\ulcorner S \equiv F\left({ }^{(} S^{\prime}\right)\right\urcorner$.

4. A detailed proof of the consistency of every I-extension of SM is given in [5], Sections 8-12. Here we present only a description of the method used. The proof is by way of a syntactic reduction procedure which is an adaptation of the method of proof used in 'tree systems'. ${ }^{1} \mathrm{~A}$ 'tree' may be defined as a set of points for which the following conditions hold. 
There is an integer assigned to each point, this integer being the 'level' of the point. There is exactly one point at level one, this point being the 'base' of the tree. Each point is connected only to point(s) at the next higher or next lower level. Every point other than the base is connected to exactly one point at the next lower level and each point is connected to at most two other points at the next higher level. Points which are not connected to any points at the next higher level are 'terminal' points.

A tree for an expression $E$ is a tree which has an expression at every point, $E$ being the expression at the base of the tree.

Expressions which either contain an expression and its negation as disjuncts or else contain a disjunct of the form $\left.\Gamma^{\prime} A^{\prime} \neq^{\prime} B^{\prime}\right\urcorner$, where $A$ and $B$ are different sentences, are 'tree axioms'.

A set of 15 schematic rules is introduced (a slight simplification of the rules in [5]). The upper lines are the premises of the rules and the lower lines are their conclusions. The order of the disjuncts in an expression is considered immaterial.

In the statement of the rules $P, Q$, and $R$ are expressions ( $Q$ perhaps being void), $A$ and $B$ are sentences, $t$ and $u$ are terms, and $v$ is a variable. $P(t / u)$ results from $P$ be replacing at most one free occurrence of $t$ in $P$ by $u$, the new occurrence of $u$ being free. $P(t / / u)$ results from $P$ by replacing all free occurrences of $t$ in $P$ by $u$, the new occurrences of $u$ again being free.

$$
\begin{aligned}
\text { (4.1) (1) } \quad P \vee Q \\
\sim \sim P \vee Q \\
\text { (2) } \sim P \vee Q \quad \sim R \vee Q \\
\text { (3) } \sim P\left(v / / v^{*}\right) \vee Q \quad \text { where } v^{*} \text { is a variable which has no } \\
\sim(E v) P \vee Q \quad \text { free occurrences in the conclusion } \\
\text { (4) }(E v) P \vee P(v / / t) \vee Q \\
\quad(E v) P \vee Q \\
\text { (5) } P \vee t \neq t \\
\quad P \\
\text { (6) } t \neq u \vee P(t / u) \\
\text { (7) } u \neq t \neq u \vee P \\
t \neq u \vee Q \\
t \neq u
\end{aligned}
$$




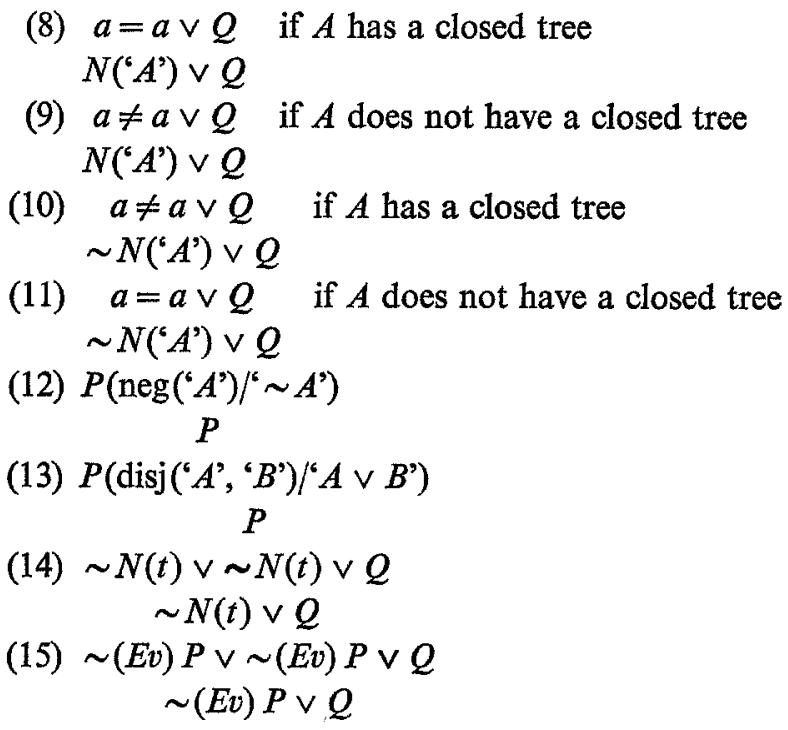

An expression is said to have a 'closed' tree if there is a tree for that expression which contains only a finite number of points, and which has a tree axiom at every terminal point, and every expression in the tree is related to the expression(s) to which it is connected at the next higher level as conclusion to premise(s) of one of the 15 rules in (4.1).

Let $\mathrm{SM}^{*}$ be an arbitrary I-extension of SM with I-axioms $\left\ulcorner a_{1}=\right.$ $\left.=' A_{1}\right\urcorner, \ldots,\left\ulcorner a_{n}={ }^{\prime} A_{n}{ }^{\prime}\right\urcorner$ and let ' $I$ ' abrreviate the disjunction $\left\ulcorner a_{1} \neq\right.$ $\neq$ ' $A_{1}$ ' $\vee \cdots \vee a_{n} \neq$ ' $\left.A_{n}{ }^{\prime}\right\urcorner$. To prove the consistency of $\mathrm{SM}^{*}$ it is sufficient to establish the following two claims.

(4.2) If $P$ is a theorem of $\mathrm{SM}^{*}$, then there is a closed tree for $r I \vee P\urcorner$.

(4.3) There is no closed tree for $\ulcorner I \vee a \neq a ?$.

The proof of (4.2) requires the specification of a set of logical axioms for SM. A standard set of such axioms is used in [5]. Both of the above claims are then proven by rather tedious inductions.

It should be pointed out that the use of the tree systems is strictly an ad hoc device for proving the consistency of SM*. The property of having closed trees does not tell us anything of an interesting philosophical nature about the expressions of SM for there are many non-theorems of SM which have closed trees. 
5. We shall begin by stating two theorems. The first is a well known theorem usually referred to as Tarski's Theorem. The second is proven by Montague ([4], pp. 156-8).

(5.1) Tarski's Theorem. If $T$ is a theory which

(a) contains $Q$ (or a relativization theoreof) as a subtheory

and also contains a one-place predicate $\operatorname{True}(x)$ such that for any sentence $A$ of $T$

(b) $\vdash \operatorname{True}(\operatorname{nr}(A)) \equiv A$

then $T$ is inconsistent.

(5.2) Montague's Theorem. If $T$ is a theory which

(a) contains $Q$ (or a relativization thereof) as a subtheory

and also contains a one-pl ace predicate $\mathrm{Nec}(x)$ such that for any sentences $A, B$ of $T$

(b) $\vdash \operatorname{Nec}(\operatorname{nr}(A)) \supset A$

(c) $\vdash \operatorname{Nec}(\operatorname{nr}(\operatorname{Nec}(\operatorname{nr}(A)) \supset A))$

(d) $\vdash \operatorname{Nec}(\operatorname{nr}(A \supset B)) \supset(\operatorname{Nec}(\operatorname{nr}(A)) \supset \operatorname{Nec}(\operatorname{nr}(B)))$

(e) $\vdash \mathrm{Nec}(\operatorname{nr}(A))$, if $A$ is a valid sentence of first-order logic then $T$ is inconsistent.

$Q$ is a subtheory of Peano arithmetic often referred to as Robinson's Arithmetic. $Q$ has the property of being finitely axiomatizable but yet sufficient for the representation of all recursive functions. A relativization of $Q$ is $Q$ with a one-place predicate in the places appropriate for interpretation as " is a number' (for details of $Q$ see [9], pp. 51ff.).

' $\mathrm{nr}(A)$ ' refers to the numeral of $T$ which, on the standard interpretation, denotes the Gödel number of $A$ under a suitable Gödel numbering. It should be noted that schemata (b), (c) and (d) of Montague's Theorem are syntactical analogues, with $\operatorname{nr}(A)$ ' in the place of SM's " $A$ ", of theorem schemata of Lewis' weakest system S1.

Tarski's Theorem can be said to be a formal representation of the antinomy of the Liar as set out in (2.1) in the sense that we can recover that antinomy by 'translating' a standard proof of Tarski's Theorem back into ordinary language. This proof involves the construction of a sentence A such that $\vdash \operatorname{True}(\operatorname{nr}(\sim A)) \equiv A$. The sentence $A$ 'says' that its own 
negation is true, or equivalently, that it itself is not true, which is just what the first sentence on $B$ in (2.1) seemed to be saying.

Since Montague's Theorem makes a point with respect to a predicate to be interpreted as '___ is necessary' which is similar to the point which Tarski's Theorem makes for a predicate to be interpreted as " is true', an obvious thought is that we should be able to 'translate' the proof of Montague's Theorem back into ordinary language to obtain some sort of modal antinomy analogous to the Liar. Indeed Montague does feel that this theorem has less than pleasant consequences for the advocate of a syntactical view of modality.

Thus if necessity is to be treated syntactically, that is, as a predicate of sentences, as Carnap and Quine have urged, then virtually all of modal logic, even the weak system S1, must be sacrificed.

This is not to say that the Lewis systems have no natural interpretation. Indeed, if necessity is regarded as a sentential operator, then perfectly natural model-theoretic interpretations may be found ... which satisfy all the Lewis systems S1-S5.

... It seems at present doubtful that any philosophical interest can be attached to S1-S4. The natural model-theoretic treatment gives a system stronger than all of them, and no satisfactory syntactical treatment can be given for any of them. ([4], p. 161)

In [2] Kaplan and Montague argue that the effect of a theorem similar to Montague's Theorem for syntactical treatments of epistemic modality is to show that some technique such as stratification into different levels of language, a popular way of avoiding the antinomies surrounding '__ is true', must also be adopted in formal treatments of syntactical epistemic modalities. ${ }^{2}$ By his claim that there can be no satisfactory syntactical theories analogous to the Lewis systems Montague presumably means that there can be no such theories which do not adopt a device like stratification into language levels. ${ }^{3}$

An obvious difference between the theories shown to be inconsistent by Montague's Theorem and theories such as SM lies in the method used for forming names of sentences. The theories meeting the antecedent conditions of Montague's Theorem use Gödel numbers as names of sentences while SM forms such names by enclosing sentences in single quotation marks. Montague is, however, quite clearly committed to the view that his theorem does have consequences for theories which form. names of sentences in the manner of SM.

... we suppose syntax to be arithmetized and use as syntax languages those languages which contain the symbols of arithmetic .... ([4], p. 158) 
We can associate with each expression a term of $Q$ which can be regarded as the standard name of that expression; to be specific we associate with the expression $s$ the name ['nr $\left.(s)^{\prime}\right]$.... ([4], p. 156)

This approach is by no means essential, and is adopted only to allow us to build on terminology and results already present in the literature. An equivalent and perhaps more natural approach would employ a syntax language ... which speaks directly about expressions. ([4], p. 158n)

It is ... desirable to introduce a system of names of expressions. Thus if $E$ is any expression, $\bar{E}$ is to be the standard name of $E$, constructed according to one of several alternative conventions. We might, for instance, construe $\bar{E}$ as the result of enclosing $E$ in quotes. Within technical literature a more common practice is to identify $\bar{E}$ with the numeral corresponding to the Gödel-number of $E$. As a third alternative, we could regard $\bar{E}$ as the structural-descriptive name of $E$ (within some well-determined metamathematical theory). A foundation for our later arguments could be erected on the basis of any one of these conventions. ([2], p. 80)

By 'structural-descriptive names' Montague is referring to a system of naming in which the name of a compound expression is a concatenate of the names of the symbols in the expression. If 'cee', 'aye', and 'tee' named ' $c$ ', ' $a$ ', and ' $t$ ', respectively, and '*' represented the concatenation function, then the structural-descriptive name of 'cat' would be 'cee*aye*tee'.

It is well known that concatenation theories are as powerful as arithmetic theories (see [3], [6]), so we have no reason to question Montague's claim that results obtainable by the use of Gödel numbers are also obtainable by the use of structural-descriptive names. Our interest is in the claim that results obtainable by the use of Gödel numbers are also obtainable by the use of quotation mark names.

6. Montague's claim is that there can be no satisfactory (unstratified), consistent syntactical modal theory which forms names of expressions by enclosing them in quotation marks and which contains the analogues of the theorems of S1. There is an apparent conflict between this claim and the fact that SM is a consistent, unstratified syntactical theory which forms names with quotation marks and contains as theorems the analogues of the even stronger Lewis system S5.

In the case of Tarski's Theorem we saw that a standard proof of that theorem involved the construction of a sentence $A$ such that $r$ True $(\operatorname{nr}(\sim A)) \equiv A$. Since $A$ could be taken as 'saying' that it is itself was not true, it was easy to construct the analogue of $A$ in a theory which used quotation mark names. This was done for ST in (3.7).

When we look at the proof of Montague's Theorem we see that it 
involves the construction of a sentence $B$ such that $\vdash \operatorname{Nec}(\operatorname{nr}(S \supset \sim B)) \equiv$ $\equiv B$, where $S$ is a conjunction of the finite axioms of $Q$. Thus the finite axiomatizability of the arithmetic subtheory seems to be essential to the proof of Montague's Theorem while it is not essential to the proof of Tarski's Theorem. If we are to reconstruct the sentence $B$ either in ordinary language or in SM, we must find some analogue for the axioms of $Q$.

Since self-reference can be achieved via a Gödel numbering, a first thought is that the axioms of $Q$ might function simply as a device for effecting self-reference. Self-reference is effected in SM by the use of I-axioms, so perhaps some set of I-axioms might correspond to the axioms of $Q$. The attractiveness of this line of thought increases when it is noted that the proof of Montague's Theorem does not assume that the axioms of $Q$ are necessary. This parallels SM's restriction against applying the necessitation rule to I-axioms. But, as it turns out, every I-extension of SM is consistent so the axioms of $Q$ must serve a purpose which can not be captured by I-axioms.

It is emphasized in both [2] and [4] that an arithmetic subtheory may serve as a syntax language, that is, as a language in which certain syntactical truths may be expressed.

By elementary syntax we understand a first-order theory containing ... all standard names (of expressions), means for expressing syntactical relations between, and operations on, expressions, and appropriate axioms involving these notions. The form of such a theory will of course depend on the convention adopted for the assignment of standard names. If the [use of Gödel numbers] is adopted, we could identify elementary syntax with Peano's arithmetic ... or even with the much weaker theory $Q \ldots$ in either case, however, supplemented by the special formulas mentioned above. ([2], p. 89n)

Montague would presumably say that the reason his theorem does not imply the inconsistency of SM is that SM does not contain enough syntax. The same criticism applied to our characterization of a modal antinomy in Part 2 would be that our notion of a modal antinomy was too narrow. There we were trying to see if a contradiction could be inferred from certain identity statements and instances of modal schemata. What we should have done is to see if a contradiction could be inferred from the identity statements, the instances of the modal schemata, and certain syntactical truths.

It is true that SM can not express a great deal of syntax. The provable syntactical truths are limited to statements of the identity and nonidentity of sentences and to statements to the effect that truth-functional 
compounds are or are not compounds of such-and-such components.

Suppose, for example, that SM were extended by allowing the single quotation mark to form terms from all expressions, instead of just from sentences, and suppose that a function symbol 'diag' were introduced to represent the diagonalization function on predicates. We might then take as 'D-axioms' all true diagonalizations such as ' $\operatorname{diag}\left({ }^{\prime} N(x){ }^{\prime}\right)={ }^{\prime} N\left({ }^{\prime} N(x)^{\prime}\right)$ ". Extending SM by D-axioms would certainly increase the amount of expressible syntax, but it is far from clear that such an extension would be inconsistent. What is required for an analogue of Montague's Theorem to hold for an extension of SM is not just that a good deal of syntax be expressible in it, but also that this syntax be finitely axiomatizable.

The possibility of a finite axiomatization of the syntax of a theory which forms names by means of quotation marks is dim. Such a theory will have an infinite number of names of expressions and, given our conventions on the referential opacity of quoted expressions, each of these names will be an irreducible surd. There seems to be no single sentence, for example, which would yield all D-axioms as deductive consequences within the theory. Quotation mark names thus are quite different from numerals and structural-descriptive names in that the latter are referentially transparent and are constructed by performing a finite number of operations on a finite number of initial symbols.

Since we form names of expressions in ordinary language by enclosing them in quotation marks it may appear that we are committed to the view that the syntax of ordinary language is not finitely axiomatizable. This seems to raise a problem as to how we could ever learn the syntax of a language like English. Actually we are committed to no such view. For when we are speaking in English about English syntax we simply ignore the referential opacity of quoted expressions and in effect treat them as structural-descriptive names.

The antinomy of the Liar shows that the artifice of treating quotation mark names as referentially opaque surds is not sufficient to prevent the antinomies surrounding the truth predicate. We must resort to additional techniques such as stratification into language levels. With the modal predicates, however, respect for the referential opacity of quoted expressions can be a sufficient safeguard against contradiction.

The University of Michigan 


\section{BIBLIOGRAPHY}

[1] Anderson, Alan Ross and Belnap, Nuel D., 'A Simple Proof of Gödel's Completeness Theorem' (Abstract), The Journal of Symbolic Logic, 24 (1959), 320.

[2] Kaplan, David and Montague, Richard, 'A Paradox Regained', Notre Dame Journal of Formal Logic, 1 (1960), 79-90.

[3] Montague, Richard, 'Semantical Closure and Non-Finite Axiomatizability', Infinitistic Methods, Pergamon Press, New York, 1961, pp. 45-69.

[4] Montague, Richard, 'Syntactical Treatments of Modality with Corollaries on Reflexion Principles and Finite Axiomatizability', Acta Philosophica Fennica 16 (1963), 153-167.

[5] Niemi, Gunnar, 'Modality and Self Reference', Ph. D. dissertation, The University of Michigan, Ann Arbor, 1970. University Microfilms, Ann Arbor, Order No. 71-4694.

[6] Quine, W. V., 'Concatenation as a Basis for Arithmetic', The Journal of Symbolic Logic 11 (1946), 105-14.

[7] Quine, W. V., 'Three Grades of Modal Involvement', The Ways of Paradox, Random House, New York, 1966, pp. 156-74.

[8] Smullyan, Raymond M., First Order Logic, Springer-Verlag New York Inc., New York, 1968.

[9] Tarski, Alfred, Mostowski, A., and Robinson R. M., Undecidable Theories, NorthHolland Publishing Co., Amsterdam, 1953.

\section{NOTES}

1 The basic principles of tree systems can be traced back to Herbrand, Gentzen, and Beth. The system most like that used in [5] is that of [1]. Further references can be found in [8].

The systems mentioned above are designed for the first-order functional calculus, although usually not with identity. The adaptation to a syntactical theory of modality is an obvious adaptation but is probably original to [5].

2 Kaplan and Montague [2] shows that a syntactical theory of epistemic modality which meets certain conditions is inconsistent. These conditions on a predicate representing "_ knows '..." are different from the conditions for the predicate $\mathrm{Nec}(x)$ in Montague's Theorem.

However, we shall take remarks made in the earlier [2] as expressing the views of the Montague of [4], since the philosophical portions of the two papers seem to be the same and are often stated in more detail in the earlier paper.

${ }^{3}$ A stratified SM would replace the single predicate symbol ' $N$ ' by an infinite number of predicate symbols ' $N_{1}$ ', ' $N_{2}{ }^{\prime}, \ldots$ and specify that $\left\ulcorner N_{i}\left({ }^{\prime} A^{\prime}\right)\right\urcorner, i \geqslant 1$, was a well-formed expression just in case $A$ was a sentence containing only occurrences of $\left\ulcorner N_{j}\right\urcorner$ for $j<i$. 